NIST Special Publication 1200-22

\title{
Challenges and approaches for particle size analysis on micrographs of nanoparticles loaded onto textile surfaces
}

Version 1.0

Samuel J. Underwood

Justin M. Gorham 


\title{
Challenges and approaches for particle size analysis on micrographs of nanoparticles loaded onto textile surfaces
}

Version 1.0

\author{
Samuel J. Underwood \\ Justin M. Gorham \\ Materials Measurement Science Division \\ Materials Measurement Laboratory
}

Samuel J. Underwood Department of Chemistry

University of Oregon

This publication is available free of charge from:

https://doi.org/10.6028/NIST.SP.1200-22

May 2017

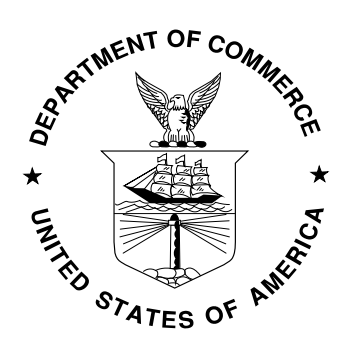

U.S. Department of Commerce Wilbur L. Ross, Jr., Secretary

National Institute of Standards and Technology Kent Rochford, Acting NIST Director and Under Secretary of Commerce for Standards and Technology 
Certain commercial entities, equipment, or materials may be identified in this document in order to describe an experimental procedure or concept adequately. Such identification is not intended to imply recommendation or endorsement by the National Institute of Standards and Technology, nor is it intended to imply that the entities, materials, or equipment are necessarily the best available for the purpose.

National Institute of Standards and Technology Special Publication 1200-22 Natl. Inst. Stand. Technol. Spec. Publ. 1200-22, 18 pages (May 2017) CODEN: NSPUE2

This publication is available free of charge from: https://doi.org/10.6028/NIST.SP.1200-22

Please direct all communication regarding this publication to the corresponding author: Justin M. Gorham (justin.gorham@nist.gov) 


\section{FOREWORD}

This National Institute of Standards and Technology (NIST) Special Publication (SP) is one in a series of NIST SPs that address research needs articulated in the National Nanotechnology Initiative (NNI) Environmental, Health, and Safety Research Strategy published in 20112. This Strategy identified a Nanomaterial Measurement Infrastructure (NMI) as essential for sciencebased risk assessment and risk management of nanotechnology-enabled products as pertaining to human health, exposure, and the environment. NIST was identified as the lead federal agency in the NMI core research area of the Strategy. This research area includes development of measurement tools for the detection and characterization of engineered nanomaterials in nanotechnology-enabled products. Textiles containing silver nanoparticles are consumer products of great interest for nanotechnology environmental, health, and safety (nano-EHS) research and for product regulation. Detection and characterization of these nanomaterials is a focus of this SP.

The protocol in this SP describes challenges and approaches for acquiring and analyzing microscope images of nanoparticles to obtain particle size distributions. An example methodology is also proposed, which is designed to be high throughput and internally consistent while limiting the number of subjective choices. While this protocol was developed with the silver nanoparticleloaded textile samples in mind, the strategies described herein could be applied to particles of any size, and the required micrographs could be gathered via scanning electron microscopy (SEM), transmission electron microscopy (TEM), light microscopy, or other image-based techniques. 


\section{Introduction}

This special publication is the result of an effort to develop high-throughput image acquisition and analysis protocol capable of reproducibly characterizing the distribution of silver nanoparticles (AgNP) on a textile by electron microscopy. This is in follow-up to a previous study, which developed a protocol for making silver nanoparticle-loaded textile test materials to be used for measurement validation ${ }^{3}$. Ultimately, this goal is best accomplished by modifying the surface of a nano-enabled textile sample, taking tens or hundreds of micrographs, and subjecting them to automated particle-sizing software routines. The method in this document relies on free opensource image processing package Fiji (Fiji Is Just ImageJ) ${ }^{4}$.

Fiji is an open source distribution of ImageJ, developed by the National Institutes of Health (NIH) (Bethesda, MD) ${ }^{4-5}$ and can be downloaded from http://imagej.net/Fiji. The procedure outlined herein uses Fiji to organize, process and analyze 8-bit images in TIFF format to calculate pixel area values for regions of interest defined by intensity thresholding after performing functions including noise correction, contrast manipulation, and spatial calibration to furnish digital images with a physical measurement scale. It is important to note that this is intended to be a guide towards developing streamlined image analysis approaches for a given sample set, and the settings employed for the AgNP example may not work for other examples which employ different samples, substrates and equipment.

\section{Materials}

The AgNP-loaded textiles in this study were prepared by a modified version of a previous study ${ }^{3}$, ${ }^{6}$. Briefly, $\mathrm{Ag}^{+}$was reduced in the presence of cotton threads at elevated temperatures to generate $30 \mathrm{~nm}$ to $50 \mathrm{~nm}$ AgNPs, in-situ. For this special publication, cotton fabric was used to prepare samples. A vial of RM 8017, the $75 \mathrm{~nm}$ AgNP reference material stabilized by polyvinyl pyrrolidone, was also used to prepare control samples.

\section{Collecting Images for Particle Size Analysis}

\subsection{Image acquisition}

The micrographs were collected with an FEI Quanta 200 field-emission environmental scanning electron microscope (E-SEM) operating in high-vacuum mode. Most images had a horizontal field width (HFW) of 3.2 micrometers and a horizontal resolution of 2048 pixels (px). Prior to imaging, samples were affixed to aluminum stubs using carbon tape, then coated with carbon in a Cressington 208 Carbon Coater using high-purity graphite rods. Images were acquired using a secondary electron detector at a working distance of $10.2 \mathrm{~mm}$ with a nominal electron beam spot size setting in the software of 3.0 or 3.5 and using an accelerating voltage of $5 \mathrm{kV}$ or $10 \mathrm{kV}$, respectively. While it is not possible to quote the exact size of the electron beam employed since we did not measure appropriate controls using these settings, the FEI provided values diameters using comparable conditions allowing us to estimate values of $2.1 \mathrm{~nm}$ and $<3.4 \mathrm{~nm}$, respectively. 

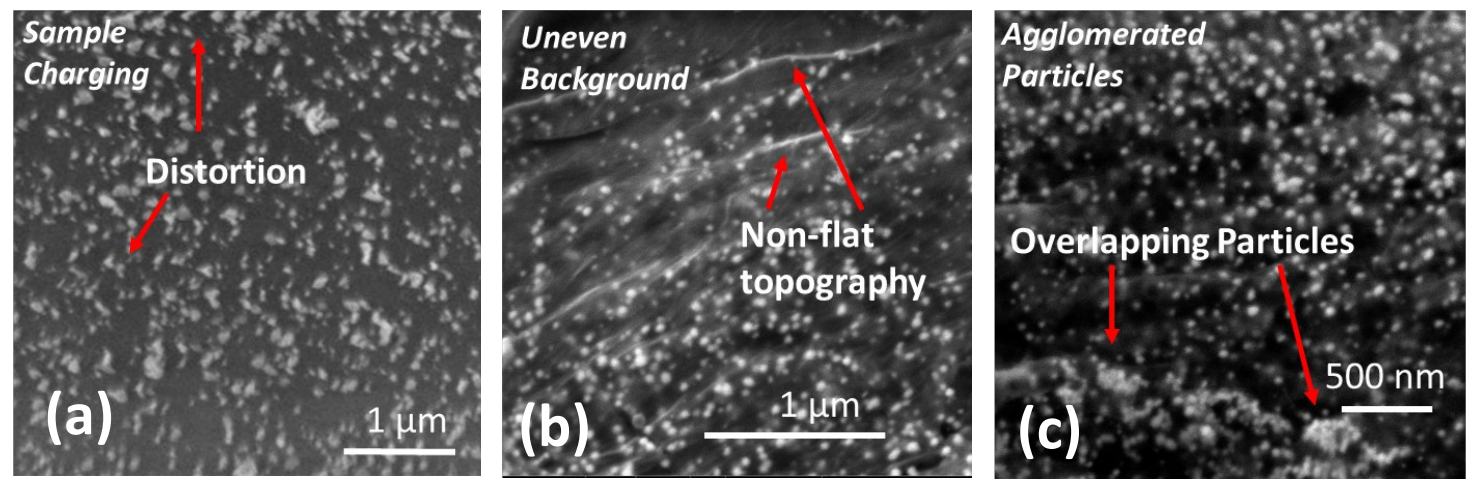

Figure 1: Examples of SEM imaging artifacts observed during the analysis of AgNP-loaded textiles. These include (a) charging, (b) edge/background effects, and (c) agglomerates and aggregates.

\subsection{Challenges}

There are inevitable challenges in acquiring representative sets of images on the varying surface of the AgNP-loaded textile, as well as in efficiently and effectively obtaining meaningful size distributions. The following three topics were found to be the primary problems in SEM image acquisition while the fourth also pertains to image analysis.

\subsubsection{Charging and distortion}

Sample charging was found to cause several artifacts including distortions and/or obscuring sample features due to the insulating textile background which was not always sufficiently conductive even with large surface loadings of AgNP or carbon coating. The second problem is that higher resolution imaging exhibits more charging due to the increased flux of electrons. An example of the charging is shown in Figure 1 (a). This charging is a problem, especially for sizing, since even a small amount of distortion will influence the accuracy of any particle size measurement. Charging can be compensated—to an extent—by modifying the surface with a conductive coating (as was done in this project) and/or by choosing optimal electron beam parameters. However, the conductive coating adds an unknown (small) amount of material to the particles and changes their size.

\subsubsection{Variable background}

Non-flat image backgrounds may occur from surface topography and charging inhomogeneity, which appear as bright (high secondary electron intensity) features resembling points and steps on micrographs (See Figure 1(b)). These features, sometimes refered to as edge effects, are problematic when trying to perform automated particle size analysis, as they may 1) overlap with existing particles, 2) be incorrectly identified as particles, 3) increase particle size or 4) completely obscure relevant features. Macros/scripts can be used to compensate to an extent for these features, and will be discussed in Section 4. However, some degree of undercounting may occur when applying macros/scripts to compensate for varying background.

\subsubsection{Agglomerates and aggregates}

Aggregates and agglomerates, as can be observed in Figure 1(c), are also found on textiles loaded with AgNP and are an interesting addition to the surface morphology. Aggregates are generally assumed to be related to the preparation of the textile. However, they do not reflect individual nanoparticles and if one is trying to determine the particle diameter of individual 
AgNPs, aggregates must not be measured. Macros/scripts can be used to compensate for these features as will be discussed in Section 4 and in the appendix, with a focus of how to exclude aggregates in the single particle size distribution determination.

\subsubsection{Efficiency versus control}

While efficiency and control are not always problems related to image acquisition or processing, these issues can be at odds when trying to ensure that measured distributions of particle sizes are quantifiably reproducibly. With respect to image analysis, two extreme approaches could be employed to estimate the particle size: 1) use a software package to manually measure each individual particle by, for example, drawing a line across each individual particle (control is maximized here) and 2) use a software package which allows for fully automatic measurement of all particle sizes (efficiency is maximized here).

There are pros and cons to each approach. For example, a fully manual measurement allows the user to have increased certainty that all measurements are of the particle in question for a handful of particles. However, by using a manual approach, it could take days to obtain a reasonable number of measurements (100's or 1000's), and there could be day-to-day variations in how the measurements are made due to small subjective user choices and user fatigue. In contrast, a fully automated approach typically requires the input of a set of parameters that the software then employs to automatically screen out artifacts and take a measurement of particles based on segmentation of each image. Thus, a fully automated approach can result in an entire image of particles sized in a matter of minutes, quickly yielding results that are consistent. However, depending on the sample and the imaging conditions, this approach could omit particles that should be included, include artifacts that should not be included, and incorrectly identify particle boundaries because of subjective choices in the coding/script commands.

In this SP, we present an approach using a combination of automated processing with manual screening, allowing for user evaluation of the particle boundaries and imaging artifacts (control) while still quickly automating many of the processing decisions into a 'one click' approach (efficiency and consistency).

\subsection{Image requirements}

Images best-suited for processing in the manner detailed in section 4 have a high concentration of bright, non-overlapping particles that stand out well against a dark background. Dark particles on a white background (as from a TEM image) are also acceptable. Images unsuitable for analysis exhibit: significant overlap of particles, poorly-defined particle edges, substantial background features, low contrast, distortion due to charging effects, astigmatism, excessive noise, or low resolution. Extensive background features can be removed to some extent during processing; however, we recommend capturing the cleanest images possible to minimize the impact of processing.

For the most effective use of processing with Fiji and macros, it is important to ensure that all imaging conditions are constant. In the authors opinion, once unsuitable images are screened out the most important variables to be kept constant are image size, pixel density, and beam conditions 
(e.g., current and voltage). Keeping these variables constant ensures measurements all have the same error and limits with respect to the size range studied.

Lastly, the image analysis method in the following section is useful to generate particle size distributions for multiple specimens with which one can find relative changes. If a true measure of the size is desired, a size calibration would be required using either a NIST or a commercial standard. This calibration control would need to consider the background/substrate (e.g., textiles are highly rough in this case) employed for the actual sample to be relevant.

\section{Using Fiji to Develop and Run Customized Macros for Image Processing and Particle Size Analysis}

Macros are especially well-suited to speeding up repetitive processes in Fiji, and they can call predefined functions to implement a variety of different actions with just a few lines of user-written script. Fiji also includes a Macro Recorder, which records user actions for easy creation of macros $^{7}$. Additionally, the Fiji Macro Language Programmer's Guide is a resource for the Fiji scripting language ${ }^{7}$. Macros are saved as text files and added to Fiji in two ways: first, by installing the macro manually and, second, by adding the new script to the already existing Startup Macro. An overall example of the macro used in this SP is available in Appendix A1 as well as much more detailed explanation of each individual step.

\subsection{Suggested processing to be included in a macro}

There are many processing steps and other techniques that can be used to improve image quality and allow the software to better assess sizes automatically. However, it is crucial that all steps used to process the raw images are recorded and the original raw images remain intact. This way, there is not loss of information, sometimes referred to as metadata, that is included by the manufacturers software on the image file. Some of the steps may be added to an image analysis scripts and some must be performed by the analyst. These steps can be separated into categories including image organization, image processing, image segmentation, and particle analysis.

\section{Image organization}

- Create a working copy of the original raw images. Store the original image set and perform all subsequent image processing on the working copy. Sometimes it is ideal to create a new folder for the processed images

- $\quad$ The first step is to define the image size, both physically and the number of pixels so, that the particles observed can be measured well. This is a step can easily be added into the script if one knows the horizontal field width (HFW), as is shown in Appendix A1.2.

- Image cropping (Appendix A1.3) is necessary to remove the metadata (bottom Figure 2(a)), since the software does not differentiate among image pixels and text pixels. A script can be modified to tune the cropping macro to one's specific images. NOTE: Do not save over the raw data file after this point or you will lose the cropped metadata permanently.

Image processing

- Apply noise and several smoothing filters (Appendix A1.4). This will help to eliminate artifacts such as pixel noise from being reported as individual particle events. 

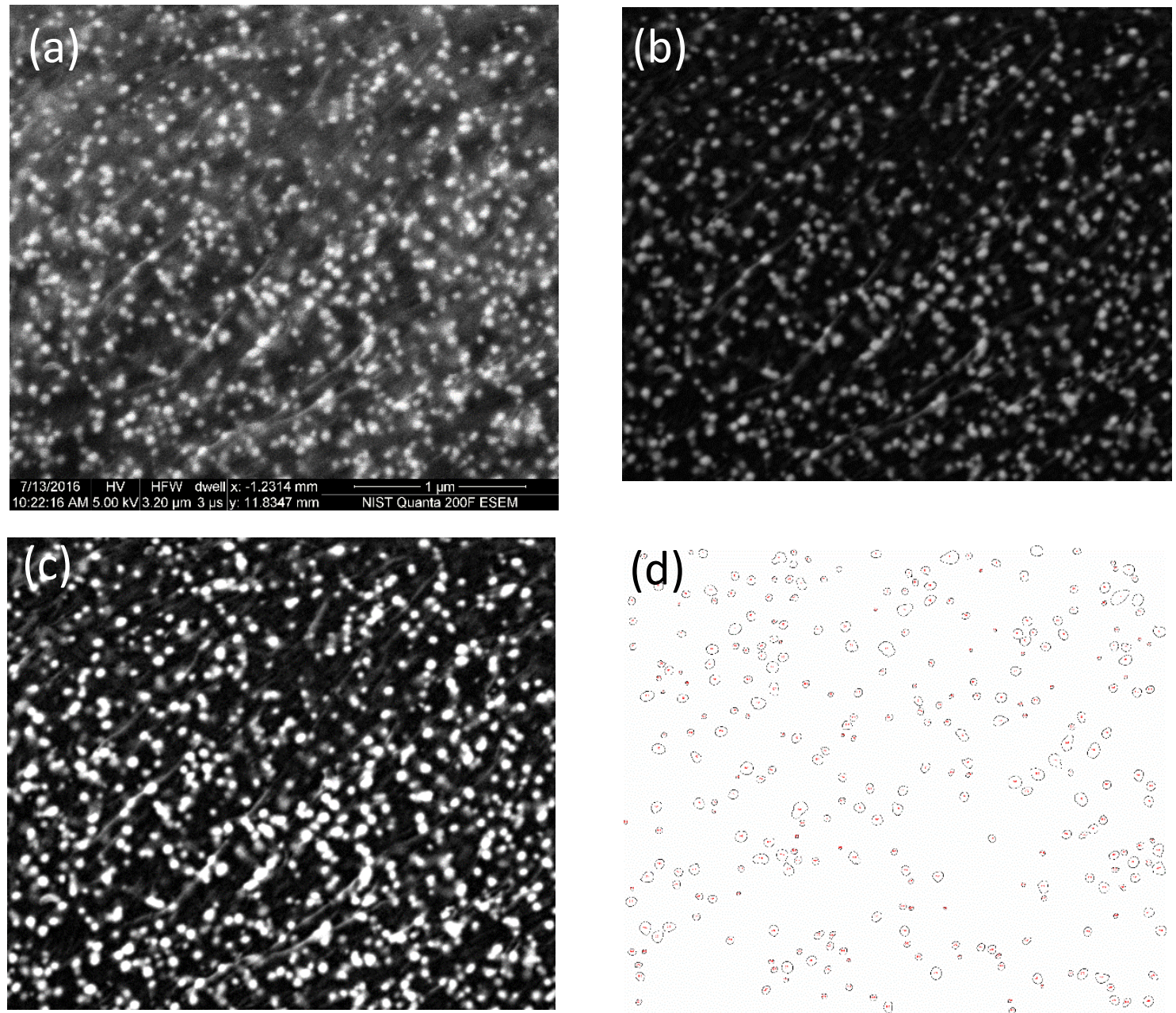

Figure 2: Demonstration of the evolution of the image during image processing. (a) Original image saved from the microscope (b) Image after cropping out metadata, scaling, noise removal and background subtraction (c) image after contrast adjustments and ready for thresholding to generate a mask, and (d) particles are identified that are consistent with the size and shape constraints that are set.

- Background subtraction can address noise that was not already corrected, as well as some other background unevenness such as edge effects that are present because of the substrate and not the particles. This is automatically done in the script shown in Appendix A1. The image in Figure 2(b) is a good example of how an image can be modified by following the steps to this point that we have outlined.

- The brightness and contrast adjustment is useful to maximize the dynamic range of pixel intensity on the greyscale (Figure 2(c)) for ease of viewing. Pixels can have a range from 0 to 255. In our script (Appendix A1.5), a dialog box automatically opens with the range adjusted. The process then automatically pauses until the user reviews, corrects and/or approves the dynamic range chosen.

Image segmentation

- $\quad$ Thresholding is one of the last steps and is used to segment the image and to make a binary mask with each pixel identified as either a 1 or a 0 , particle or background. A greyscale intensity cutoff is assigned to generate this binary mask which can be automatically 
determined by Fiji or determined by the analyst. For our script, we have chosen to include user input by automatically thresholding but requiring approval or modification before proceeding (Appendix A1.5) ${ }^{8}$.

\section{Particle analysis}

- Circularity is a value used to screen the particles that would otherwise be counted for abnormal shapes and aggregates. This is calculated for each particle automatically based on an adjusted ratio of the area: perimeter ${ }^{2}$ that is set between 0 and 1 , with 1 being a perfect circle (Appendix A1.6). User input is required to set the circularity in the script that we have written in Appendix 1.

- A size minimum allows the removal of noise that was not accounted for in earlier steps. In our script, user input is required to set the size minimum.

- Particles on the edge of the image should not be included because their size is unknown. We have included this automatically in our script.

- The final step is to calculate the size of the particles. Fiji defaults to area of the particle and provides a spreadsheet/table of areas for each particle based on specifications set in 'Image organization'. Additionally, a series of outlines are also provided in our script (See Figure 2(d)) which are representative of the particles provided in the spreadsheet that have met the user defined circularity and size requirements. This outline is important to screen out non-particles (section 4.3).

The utility of this method, especially in scenarios with more than one specimen, will greatly depend upon the quality and consistency of the correction of the noise and segmentation of the image. This is because small variations between images can result in large size changes, especially
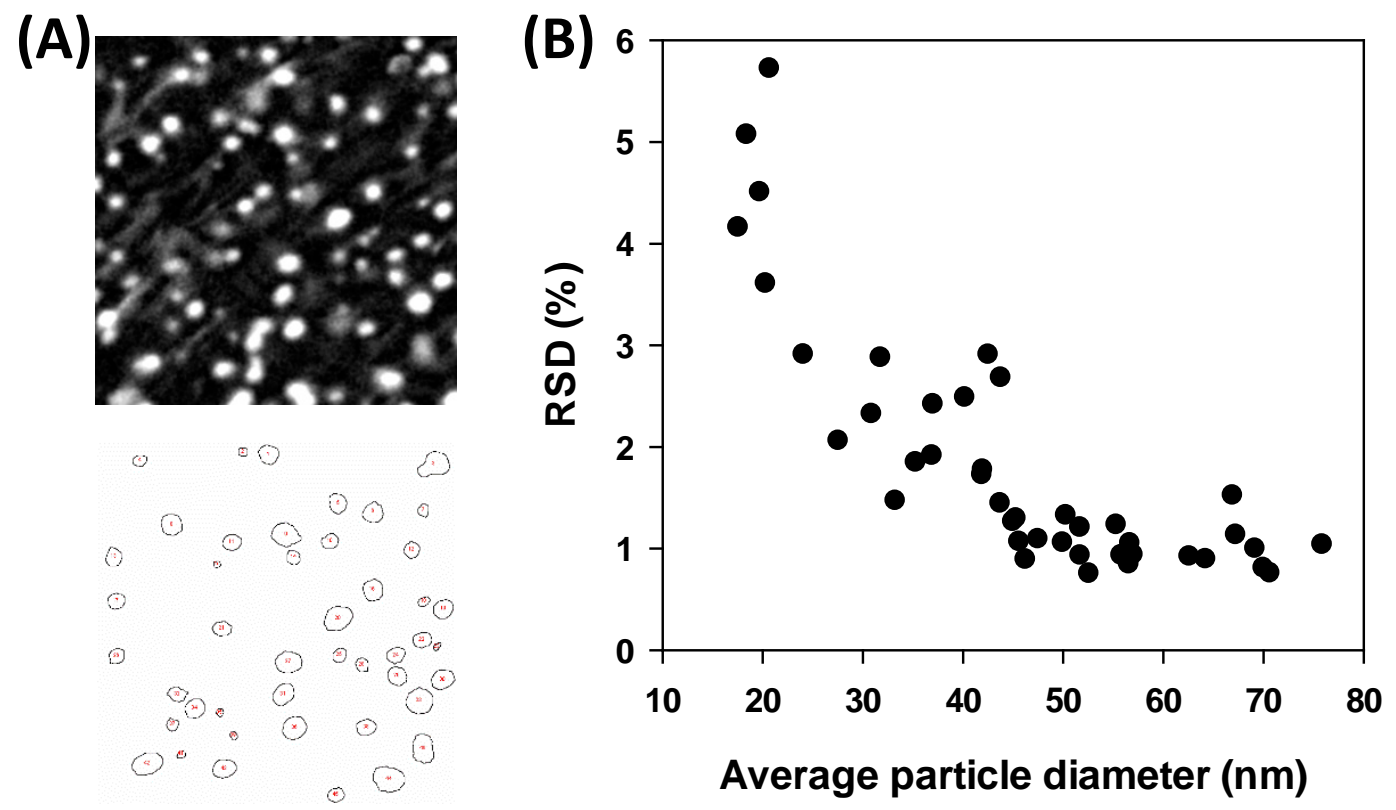

Figure 3: Impact of small changes in processing on average particle diameter. (A) Small section $(1 \mu \mathrm{m} \times 1 \mu \mathrm{m})$ of Figure 2 and representative mask of outline of pixels for acceptable particles. (B) The impact of changing the threshold step on particle diameter by plotting the RSD as a function of average particle diameter with pixel boundaries by 7 different sequential set points. 

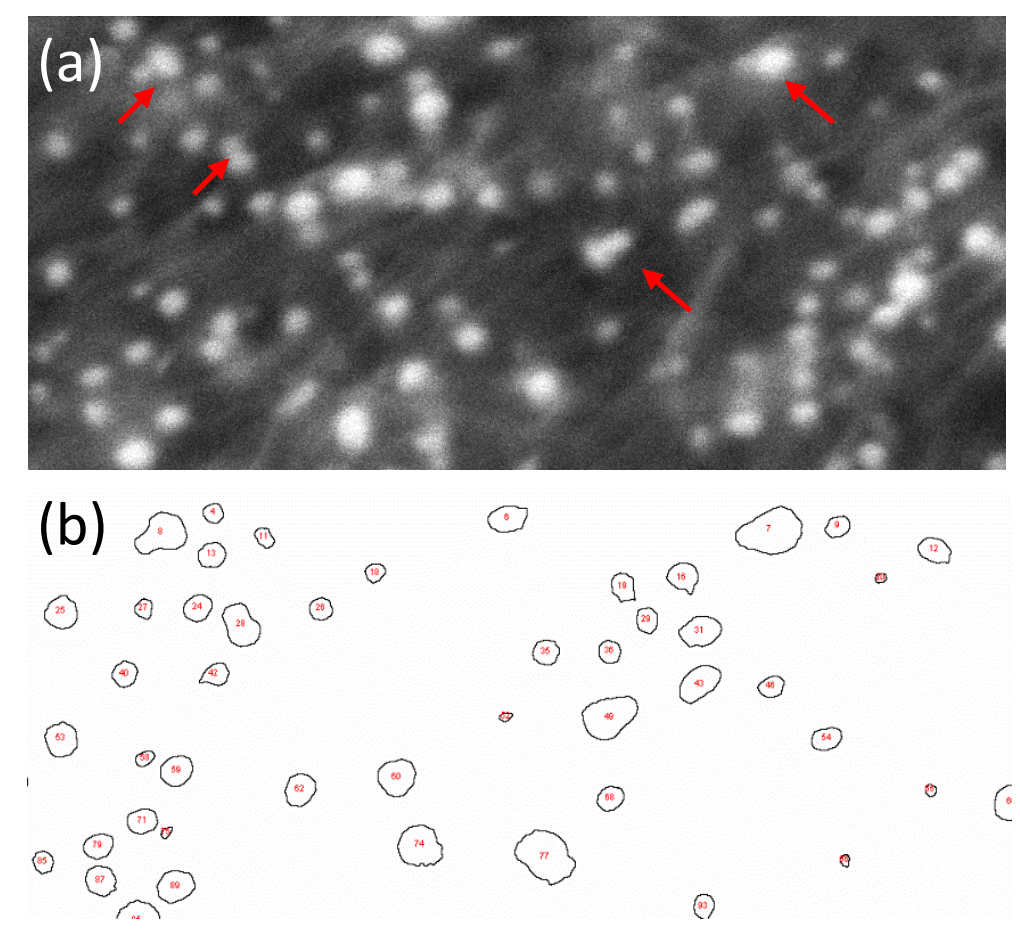

Figure 4: (a) Section of the image from Figure 2(a) prior to processing. Arrows indicate adjoining particles which are included as single particles in (b) which represents the particle included with a 0.75 circularity minimum.

\footnotetext{
particle diameters since comparable changes in absolute pixelc
}

on smaller particles.

\subsection{Sources of variability}

The user should be aware that there are several points where measurement uncertainty can arise and work to minimize it. As mentioned in the previous section, consistent criteria for assignment of the contrast and the threshold are essential to obtaining sizes across different images that can reliably be compared to one another. Other sources of uncertainty are the HFW, or imaging size, and the pixel density set for the samples characterized. Both relate to the minimum particle size based on the pixel limits set that can be resolved. If images used are acquired under different HFWs or pixel densities, the results may be skewed to average particle diameters of a smaller value.

\subsection{User input to eliminate particles that are not screened out by the macro}

While in an ideal case a one click/one command prompt would produce useable results from raw micrographs that have zero flows, it is not a realistic expectation. The heterogeneous nature of the textile surface, requires different corrections in each image. Furthermore, there is the possibility for adjoining particles (see Figure 4) and aggregates not to be screened out with the processing, so one must review and remove particles that are not reflective of the desired measurement. It is essential that the criteria for removing a particle is kept consistent. 


\subsection{Calculating particle diameter and generating a histogram}

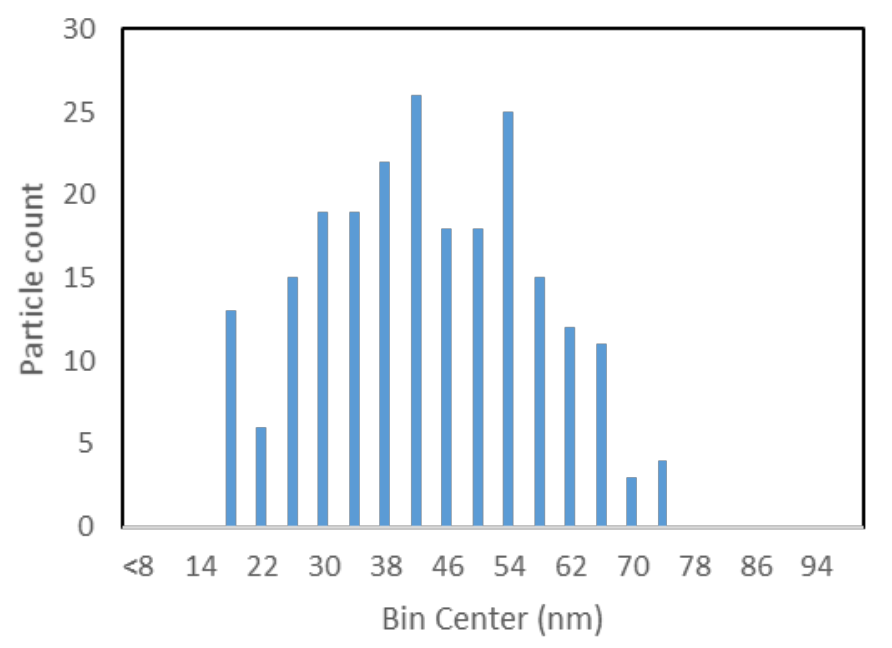

Figure 5: Histogram of particles in the image of Figure 2(a) after processing with the macro in Appendix A1.

Upon completion of the particle analysis in section 4.1, a spreadsheet including a list of particle areas are provided based on the size minimum and circularity limits set. From this list, the user can calculate a diameter (d) based on the measured area (A) from the spreadsheet reflective of a circle of equivalent area, no matter what shape it appears on the micrograph using the following equation:

$$
d=2 \sqrt{\mathrm{A} / \pi}
$$

Always double-check that Fiji has printed the results table in the units appropriate. Figure 5 is a histogram representative of the distribution of particles from the image in Figure 2(c) using a 5-pixel radius minimum size and a value of 0.85 for the circularity. The average particle diameter was $43.5 \mathrm{~nm}$ $\pm 13.9 \mathrm{~nm}$. Due to the low number of particles analyzed, this histogram is merely meant as a tool for illustration and not necessarily an accurate reflection of the sample. It is important to note that with a 5-pixel minimum, there will be a $15.6 \mathrm{~nm}$ cutoff on particle diameter for the image size and pixel density. For measurements of small-size particles represented by only a few pixels, higher resolution imaging may be required.

\section{Control to Check Particle Analysis Procedure: RM 8017- 75 nm AgNPs}

To demonstrate the effectiveness of the use of the approach in section 4 using Fiji, a control was devised using the $75 \mathrm{~nm}$ silver nanoparticle reference material (RM 8017) ${ }^{1}$. While this control does not include a substrate with comparable properties to the cotton fibers (e.g., roughness, charging), it does benefit from having known values for size attributed to the actual material that we are characterizing, which is important when assessing the technique for viability.

\subsection{Experimental details}

RM 8017 was reconstituted in accordance to instructions in the certificate and drop-cast onto a Nanoplus TEM grid from Dune Scientific ${ }^{1}$. Secondary electron micrographs were collected with an FEI Quanta 200 field-emission environmental scanning electron microscope (E-SEM) operating in high-vacuum mode, with a working distance of $10.2 \mathrm{~mm}$, a spot size of $2.5 \mathrm{~nm}$, and an accelerating voltage of $10 \mathrm{kV}$. Magnification was set to produce images with a horizontal field width of $3.20 \mu \mathrm{m}$ and a horizontal resolution of 2048 pixels. 

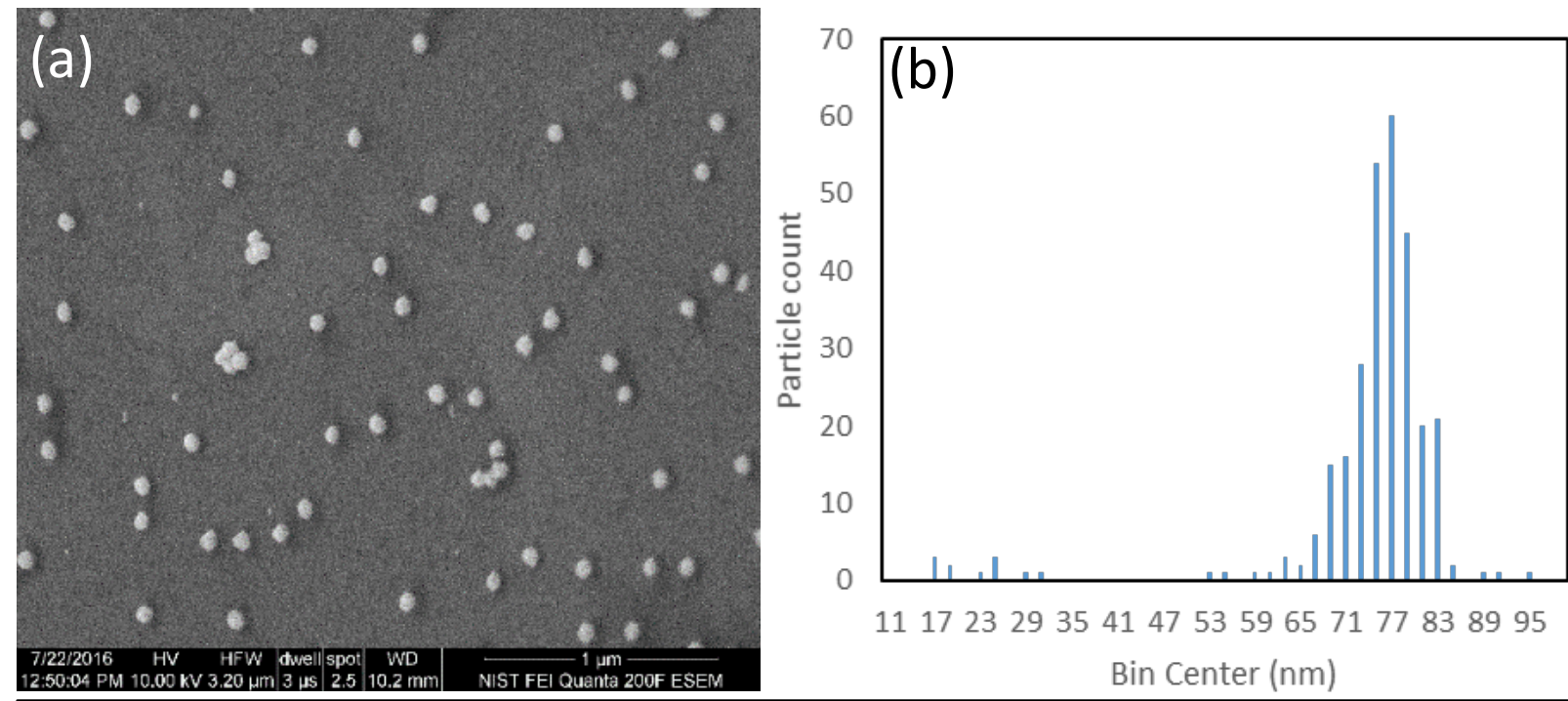

Figure 6: (a) Representative images of RM 8017, nominally $75 \mathrm{~nm}$ silver nanoparticle reference material before image processing. (b) Histogram of particle count vs. diameter over the range of particles measured and presented in Table 1 . A total of 290 particles were counted from 13 total images.

\subsection{Image processing in Fiji}

Micrographs acquired, such as the one shown in Figure 6(a), were subjected to processing in Fiji outlined in section 4.1 and further explained in appendix A1.

1. Crop: The data bar at the bottom of micrographs was removed.

2. Using the macros discussed in section 4.1, a rolling ball background subtraction was applied with a ball radius of 40 pixels, a smooth function was applied five times, and a despeckle function was applied once.

3. In this case, the threshold was manually adjusted to ensure the whole particle was accounted for in the analysis.

4. Maps were created for visual inspection and false positives (aggregates and artifacts) were removed.

Table of RM8017 values

\begin{tabular}{|c|c|c|}
\hline & $\frac{\text { SEM value }}{\text { with script }}$ & $\frac{\text { TEM ref. }}{\text { value }^{\mathbf{x}}}$ \\
\hline \# of particles & 290 & $>16000$ \\
\hline Average $(\mathrm{nm})$ & 73.9 & 74.6 \\
\hline St. Dev $(\mathrm{nm})$ & 11.4 & 3.8 \\
\hline Max $(\mathrm{nm})$ & 96.0 & N/A \\
\hline Min $(\mathrm{nm})$ & 16.3 & N/A \\
\hline Median $(\mathrm{nm})$ & 76.3 & N/A \\
\hline
\end{tabular}

Table 1: Values and statistics from the particle size analysis in FIJI from images of RM 8017 silver nanoparticles based on 13 images. For comparison, TEM reference values from the RM 8017 certificate are provided ${ }^{1}$.
5. The resulting data is plotted in a histogram in Figure 6(b) and a list of values extracted from this processing are in Table 1.

Table 1 presents some of the results obtained from the measurements of this sample. The top three values are likely the key pieces of information from the 13 images processed, segmented and analyzed. The average and standard deviation allows for a rough comparison with the established TEM reference values. The $73.9 \mathrm{~nm}$ circular equivalent measurement result by the SEM, obtained from a much smaller number of particles, is indeed well within the error associated with TEM. The reason 
why the uncertainty is higher for the SEM measurement is the small number of particles measured and that the TEM value represents the average and standard deviation of 9 separate vial of RM 8017 (the average or the averages). ${ }^{1}$ Regardless, while one could obtain better statistics by measuring more particles, this still provides another good demonstration on how high through-put processing using macros in Fiji can be employed to analyze particles from a set of images.

\section{Abbreviations}

AgNP - Silver nanoparticles

e-SEM - Environmental SEM

Fiji - Fiji is just Image J

HFW - Horizontal Field Width

Nano-EHS - Environmental, health, and safety research as it pertains to nanomaterials

NIH - National Institute of Health

NIST - National Institute of Standards and Technology

NNI - National Nanotechnology Initiative

$\mathrm{RM}$ - Reference Material

RSD - Relative Standard Deviation

SEM - Scanning electron microscopy

TEM - Transmission electron microscopy

XPS - X-ray photoelectron spectroscopy

\section{Acknowledgements}

We would like to thank Debra Kaiser for her direction and vision in the characterization of nanomaterials in consumer products from which the development of this protocol derived. SJU would like to thank the NIST Summer Undergraduate Research Fellowship program for support.

\section{References}

1. NIST RM 8017 - Polyvinylpyrrolidone Coated Silver Nanoparticles (Nominal Diameter 75 nm). https://www-s.nist.gov/srmors/view detail.cfm?srm=8017.

2. National Nanotechnology Initiative: Environmental, Health and Safety Research Strategy; Presidents Council of Advisors on Science and Technology: National Science and Technology Council Committee on Technology: Washington, DC, 2012.

3. Gorham, J. M.; Murphy, K.; Liu, J.; Tselenchuk, D.; Stan, G.; Nguyen, T. M.; Holbrook, R. D.; Winchester, M.; Cook, R. F.; MacCuspie, R. I.; Hackley, V. A., Preparation of silver nanoparticle loaded cotton threads to facilitate measurement development for textile applications. NIST Technical series pubication 2015.

4. Schneider, C. A.; Rasband, W. S.; Eliceiri, K. W., NIH Image to ImageJ: 25 years of image analysis. Nat Methods 2012, 9 (7), 671-675.

5. Schindelin, J.; Rueden, C. T.; Hiner, M. C.; Eliceiri, K. W., The ImageJ ecosystem: An open platform for biomedical image analysis. Mol Reprod Dev 2015, 82 (7-8), 518-529.

6. Lee, P. C.; Meisel, D., Adsorption and Surface-Enhanced Raman of Dyes on Silver and Gold Sols. $J$ Phys Chem-Us 1982, 86 (17), 3391-3395.

7. Mutterer, J.; Rasband, W. S., Image J Macro Language Programmer's Reference Guide v1.46d. RSB Homepage 2012, 1-45.

8. Auto_Threshold. http://imagej.net/Auto_Threshold.

9. Particle Analysis. http://imagej.net/Particle_Analysis. 


\section{Appendix A1}

The text file available from https://doi.org/10.6028/nist.sp.1200-22s is an example of a macro used in this SP for demonstration purposes. It is important to note that much of the choices made for image processing here shall be modified to fit the task at hand.

\section{A1.1 Commenting the script and defining a macro}

The authors consider it good practice to comment, or annotate, the script that one writes into the text file so future readers can understand what sections of the script are responsible for which actions. For Fiji, addition of a line for a comment requires the addition of "//" symbols before the text describing the action. This is required for each line.

Note that a script is started and finished using the following symbols: $\{$ and $\}$, respectively. Each macro is started by identifying the title of the macro and a hotkey associated with launching the macro once it is loaded into Fiji. Macros can also be launched under the "Macros" in the "Plugins" menu.

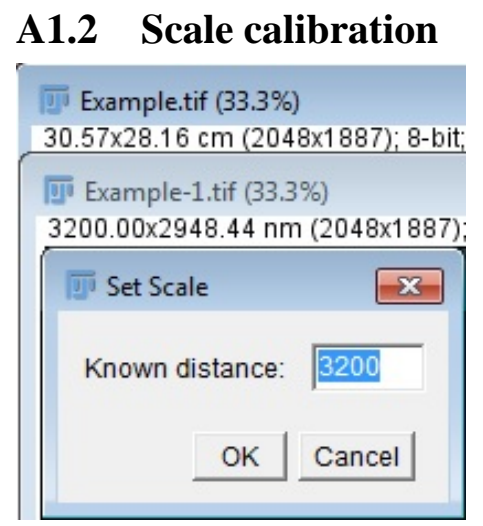

Figure A1: Demonstration of how the information on the resolution line for an image should change when the scale is calibrated.
At some point during particle sizing, a user must calibrate the scale, so that the values associated with particles from the processed images can be converted from pixel areas to areas in square nanometers. A quick scale macro can be used to speed up the "set scale" process. This is shown in step 2 of our macro, which automatically calibrates the image with a user-input horizontal field width (HFW) and extracted horizontal pixel count. Figure A1 shows the custom macro being used to calibrate an image to $3200 \mathrm{~nm}$. For high accuracy work pertinent ISO or other traceable scale calibration methods shall be used.

\section{A1.3 Automatic cropping of images}

Electron micrographs will often have metadata printed at the bottom of the image. While this data bar is useful because it contains useful information, the data bar must be removed prior to particle analysis. A "crop" macro can be installed to speed up the process of removing the data bar, such as that seen in step 3 of the main text

The current script for the crop macro reduces the image to 2048 pixels * 1765 pixels (3200 nm x $2757.8 \mathrm{~nm}$ ), removing the data bar from the bottom of the image as seen in Figure A2 (center). While this macro is sufficient to remove the metadata that our SEM software attaches to the bottom of our images, this will vary from instrument to instrument. To find the correct size for your instrument, the authors recommend selecting the menu command "Plugins-> Macros->Record...", then selecting the desired area (without the data bar) using the rectangular area selection tool. The Recorder should display "makeRectangle $(\odot, \odot, \mathrm{x}, \mathrm{y}) ;$ ". The user should use this line of script to replace/correct the first line in Step 3 and save the file for future use. The crop function can also manually be performed under the "Image" menu of Fiji. 


\section{A1.4 Correcting noise, smoothing and background subtraction}

Fiji has built-in tools to remove unevenness using a "rolling ball" background subtraction method with a ball radius of, for example, $40 \mathrm{px}$. Additionally, Fiji has tools for removing salt and pepper noise called "Despeckling" and edge jaggedness using a smoothing process ${ }^{5}$. These tools can be used manually and are found under the "Process" menu. Experimentation to determine the most appropriate set of settings is indispensable, as these vary case by case.

\section{A1.5 Brightness/ contrast and thresholding}

To perform automatic particle analysis (to find particle size), a binary image is needed, in which each pixel is either black or white ${ }^{9}$. Set a threshold only after processing the image so that particles show clearly against the background. Setting a threshold can be done manually. To do so, select "Image->Adjust->Threshold..." (Ctrl+Shift+T). Drag the sliders until all particles are filled with red, out to their edges. Figure A2 shows an image that is actively where a threshold is about to be applied. As can be observed, a grayscale histogram is displayed, and the user drags sliders to choose a threshold. Pixels shaded red will be converted to white (255), while pixels outside the red sections will be converted to black (0). This is a necessary step for particle analysis. For black particles on a white background, deselect the "Dark background" box.

\section{A1.6 Analyzing particles}

Fiji's Analyze Particles function uses the binary image created in the last step to generate a list of particle areas. Select the menu command "Analyze->Analyze Particles..."

Analyze Particles has two important parameters:

\section{A1.6.1 Size}

Size dictates the minimum particle size (area) that will be picked for analysis. A size no less than 78.5 pixel $^{2}$ was used, dictated by the 2048 pixel image resolution. Size minimum and maximum can help in excluding artifacts, agglomerates and particles that differ in size from those particles of interest.

\section{A1.6.2 Circularity}

Circularity is calculated as $4 \pi \cdot \frac{\mathrm{A}}{\mathrm{P}^{2}}$, where $\mathrm{A}$ is the area of the analyzed particle, and $\mathrm{P}$ its perimeter. A circularity value of 1 is a perfect circle.

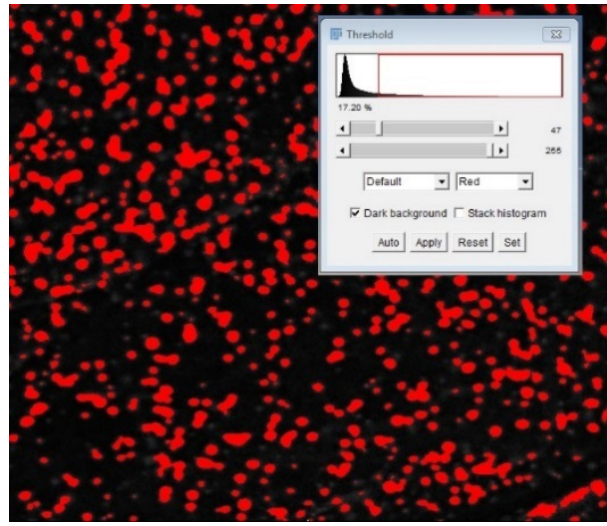

Figure A2: Setting a Threshold by Hand

\section{A1.6.3 Other settings}

The Analyze Particles function calculates the area of a particle by counting the pixels inside a section defined by a threshold, then applies the user-defined scalar to convert the pixel measurement into a spatial measurement.

Tick both "Display results" and "Clear results". This will print a list of particle areas and indices, and will clear and previous measurements. "Exclude on edges" is also recommended, as it excludes particles that are partially cut off by the edge of the micrograph.

A1.6.4 Analysis results 
Fiji will display a spreadsheet of values representative of particles in a micrograph that meet the circularity and size minimum requirements. It is recommended to save the spreadsheet either by copying it into a program such as Excel, or by saving it from the file menu as a text file. 